\title{
Personality Disorders and the Five-Factor Model Among filipino Non-Clinical Sample
}

\author{
Mary Rachelle R Wapaño, $\mathrm{PhD}$ \\ Kinaadman Research Center, Xavier University - Ateneo de Cagayan, Philippines
}

\begin{abstract}
This study aimed to explorethe relationship between the personality disorders and five factor modeland their factor structures in a Filipino non-clinical sample. The participants were 828 respondents from various areas in the Philippines. 35.5 $\%$ males and $64.3 \%$ females. The average age of respondents is $20.29(S D=4.83)$. The Millon Clinical Multiaxial Inventory (MCMI-III) and the Neuroticism, Extraversion and Openness Personality Inventory - Revised (NEO-PI R) were used as assessment measures. Eight factors were extracted from a varimax principal components analysis including the 30 NEO-PI$R$ facets and the 14 PD scales. The results show that the fivefactor model is modestly related to personality disorders as described in the DSM IV. There is a modest correspondence between personality disorder and NEO dimensions and facets. Regression analyses showed that NEO domain and facet scores explained a fifth to a third of the variance in PD dimensions. The results lend measured support to the correspondence of personality disorders and FFM theory of personality, however, the breadth of personality disorder pathology is not completely covered by the NEO. It seems then necessary to use additional clinical material, beyond the NEO, to describe personality disorder dimensions.
\end{abstract}

Key words: personality disorders, five-factor model, openness to experience, conscientiousness, extraversion, agreeableness, neuroticism

\section{INTRODUCTION}

$\mathrm{S}$ cholars in personality psychology and clinicians are currently exploring the idea that the Five-Factor Model (FFM) of personality is the most functional and inclusive taxonomy for understanding problems associated with personality disorders. This study attempts to explore the relationship of personality disorders and the FFM as well as explore the factor structures of PD and FFM in Filipino nonclinical sample.

Personality disorders may be best described by three pathological characteristics (Millon, Millon, Grossman, \& Meagher, 2002). The first comes from the view that personality is the psychological equivalent of the body's immune system: Personality disorders tend to reveal tenuous stability under conditions of stress. Whereas normal individuals change coping strategies that prove to be ineffective, personality disorder subjects tend to repeat the same coping responses which as a result increases the level of stress, creates crisis situations and distorts perception of social reality.
The second characteristic of personality disorder subjects overlies the first. Personality disorder subjects are highly inflexible and have very limited alternate coping strategies that they impose on situations where they are inappropriate. They completely control and direct social situation with the intensity and rigidity of their traits which in turn creates the strongest constraint in their interactions. The third characteristic is the result of the second: Because personality disorder subjects are incapable to adjust, the dominant pathological themes tend to repeat in vicious circles. Millon et al. (2002) posit that "pathological personalities are themselves pathogenic" (p.14). The repetitive maladjusted behaviors and self-defeating themes create new problems, waste chances for learning or improvement and generate new situations where failures are repeated.

In sum, personality disorder is characterized by chronic use of inappropriate, rigid, and maladaptive coping mechanisms.

Unfortunately, there is no consensus in the field regarding the fundamental constructs that comprise personality pathology.

Two research problems are addressed in this study: This study examined the factor structure of personality disorders and measures of the five-factor model of personality. This alsoexamined the links between personality disorder scales and the Personality disorder scales are assessed by use of the MCMI-III (Millon Clinical Multiaxial Inventory, Millon, Millon, and Davis, 1994) and the five factor model of personality is assessed using the NEO-PI R (NEO-PI-R, Neuroticism, Extraversion and Openness Personality Inventory - Revised, Costa \& McCrae, 1992).

This study investigates the relationship between the FFM and personality disorders in a Filipino non-clinical sample. Although the FFM has been found in a variety of languages, lexical studies in Filipino have general uncovered more than five factors. For example, Imperio, Church, Katigbak and Reyes (2008) identified 10 physical and social persondescriptors named as prominence, uselessness, attractiveness, respectability, uniqueness, destructiveness, presentableness, strength, dangerousness, and charisma. Additionally, Church, Reyes, Katigbak, and Grimm (1997), identified seven Filipino dimensions comparable to the Big Five model. In their further exploring the Filipino personality structure, Church, Katigbak and Reyes (1998), again identified seven personality dimensions while Mallare (2007) identified six Philippine trait dimensions, as gregariousness, concern for others, 
conscientiousness, temperamentalness, self-assurance and intellect.

It is important to note that the DSM (Diagnostic and Statistical Manual) of Personality Disorder categories may have their own cultural limitations. The DSM-IV (Diagnostic and Statistical Manual, $4^{\text {th }}$ edition) tends to represent a Western model of mental ill-health. It fails to address the various mental disorder issues that relate the meanings of illness and treatment and the expressions of distress across a diverse array of ethnic, cultural, racial groups (Mezzich\&Ustun, 2002).

In the light of the evidence from research that Filipino lexical studies identify more than five factors, and that DSM personality categories face cultural limitations, the current study is an is an exploratory study of the relationship between personality pathology and DSM diagnoses in Filipino. This is an exploratory study in that this is considered as an initial research to understand the relationship between the personality disorders and factors in a Filipino context.

This relationship is interesting to investigate as, to date, it is not clear whether either is culturally appropriate. There are no clear evidence whether the five-factor model or the DSM personality disorders are appropriate for Filipino culture. Katigbak, Church, Guanzon-Lapena, Carlota and del Pilar (2002) found that few local constructs are not very well accounted for by the Five Factor Model, and concluded that these constructs, although not unrecognized in the Western culture may be specially salient of composed differently in the Filipino context.

To date research results are not clear on whether the previous pattern of relationships found for the five-factor model and DSM personality disorders will be found in this culture

\section{LITERATURE REVIEW}

The Diagnostic and Statistical Manual for Mental Disorders (DSM IV, 2004 ) lists 12 personality disorders, 10 of which are formally recognized and two of which are provisional. The DSM IV lists personality disorders in three clusters on Axis II:

Cluster A, the Odd or Eccentric cluster includes: (a) paranoid characterized with being guarded, cynical, distrustful, and suspicious; ,hyper vigilant to the motives of others to do harm, seeking evidence for schemes, feels virtuous but persecuted; (b) schizoid who are described as indifferent, isolated, solitary; does not need or want human attachment, minimally aware of the feelings of others, if few drives or aspiration.

Cluster B is referred to as the dramatic, emotional or erratic cluster: (a) antisocial characterized by impulsivity, recklessness, acting without thought; disrespect for social customs, traditions; (b) borderline is described as being unstable, scheming; intensely fears rejection and isolation; rapid fluctuation of moods; swiftly shifts from being loving to hating; sees self and others as either wholly good or wholly bad; (c) histrionics are dramatic, seductive, shallow, seductive, vain; seductive to minor events, exhibitionistic to secure notice; sees self as attractive and charming; and (d) narcissistic personality disorder is characterized as egotistical, arrogant, grandiose; preoccupied with fantasies of success, beauty, and achievement; sees self as admirable and thus entitled to special treatment.

Cluster $\mathrm{C}$ is referred to as the anxious or fearful group: (a) avoidant is characterized as hesitant, self-conscious, embarrassed, anxious and is tense in social situations; (b) dependents feel powerless, incompetent, submissive, immature; withdraws from adult responsibilities; seeks constant comfort from stronger figures; (c) obsessivecompulsive is described as being restrained, meticulous, respectful, inflexible; lifestyle is rule-bound; adheres to social conventions; sees world in terms of hierarchy and regulations, sees self as committed, dependable, efficient and productive.

These personality disorder constructs in the Axis II of the DSM IV were derived from clinical experience and diagnostic committee consensus (DSM IV, 2004).Some clinicians find these diagnoses useful for describing particular forms of patient difficulties while other practitioners identify other patient difficulties other than the current Axis II categories.

Scholars in personality psychology and clinicians are currently exploring the idea that the FFM of personality is the most functional and inclusive taxonomy for understanding problems associated with personality disorders. The FFM is a hierarchical model personality that was originally inspired by research in the lexical tradition. The lexical hypothesis proposed by Galton in 1884 (Mondak, 2010) holds that all the important dimensions of variation in personality are already encoded in the language. With a comprehensive sample of personality trait terms and factor analysis based on large number of respondents, the fundamental dimensions of personality can be revealed. The exact number and nature of these dimensions is a continuing source of controversy. For example, the HEXACO, which represents personality dimensions honesty, emotionality, extraversion, agreeableness, conscientiousness, and openness to experiences, is a personality model that assesses six personality dimensions found in the lexical research of personality structure conducted in various languages. To date, the personality field appears to have standardized on five broad dimensions, or factors, as fundamental and universal to personality. The five factors are Neuroticism, Extraversion, Openness to Experience, Agreeableness, and Conscientiousness (Costa \&Widiger, 2002).

As operationalized by the NEO-PI-R, each factor is further described by six more specific traits, known as facet (Costa \& MacCrae, 1992). The FFM this consists of thirty traits.

Neuroticism (N)refers to a persistent level of emotional instability. High $\mathrm{N}$ scores suggest higher levels of high anxiety and volatility while low $\mathrm{N}$ scores suggest low anxiety and stability. N, with alternate label NA, also includes having 
improbable ideas, extreme cravings, and maladaptive coping behaviors. $\mathrm{N}$ facet scales include anxiety, hostility, depression, self-consciousness, impulsivity and vulnerabtility (Costa \&Widiger, 2002).

E for Extraversion refers to the extent and strength of preferred interpersonal relations, activity level, and need for stimulation, and capacity for joy. High E scores show that a person is outgoing, energetic, talkative, person-oriented, cheerful, and warm. On the other hand, low E scores show that a person is likely to be reserved, restrained, aloof, selfsufficient, and quiet, but not necessarily unfriendly.

O for openness involves the active seeking of appreciation of novel experiences for their own sake. Open individuals are questioning, inventive, and willing to entertain new ideas and alternative values. Closed individuals, those with low $\mathrm{O}$, tend to hold traditional values and attitudes, conservative and dogmatic in their beliefs, and set in their behaviors.

A, agreeableness, like E, is an interpersonal dimension measures how compatible people are with other people. High A scorers tend to be sympathetic, good natured, trusting, considerate, and altruistic. While low A scores tend to be hostile, pessimistic, rude, abrasive, suspicious, difficult, calculating and unforgiving.

$\mathrm{C}$, conscientiousness, refers to the extent of organization, diligence, control and drive of goal-related behaviors. High $\mathrm{C}$ individuals tend to be organized, dependable, diligent, selfdirected, motivated and persevering, while low $\mathrm{C}$ individuals tend to be directionless, undependable, negligent and selfindulgent (Costa \&Widiger, 2002). .

Many studies have been done to relate the FFM with personality disorders.Saulsman and Page (2004) performed metaanalyticstudies which involved 15 studies, different samples, and different FFM measures such as the NEO-PI-, varied clinical assessments such as different versions of the MCMI. The authors concluded that their results were consistent with the hypothesis that personality disorders can be extreme, extension of normal personality, and are maladaptive variants of the five factors (Costa, and Widiger, 1994, 2002). This study revealed thatpersonality disorders showed significant relationship with the five factor model. For example, disorders characterized by emotional distress such as Paranoid, Schizotypal, Borderline, Avoidant and Dependent, are positively linked with Neuroticism. On the other hand, disorders characterized by sociability, like Histrionic and Narcissistic disorders, were related to positively related to extraversion while disorders defined by shyness such as Schizoid, Schizotypal, and Avoidant disorders are found to be negatively associated with Extraversion. Paranoid, Schizotypal, Antisocial, Borderline and Narcissitic disorders, all of which are defined by interpersonal difficulties, showed a negative relationship with Agreeableness. Disorders characterized by orderliness such as Obsessive-Compulsive is positively related to Conscientiousness, while those defined by recklessness such as Antisocial disorder is negative related to Conscientiousness.

\section{RESEARCH METHODOLOGY}

Research Design: This is a correlational research design study. As a correlational research, this is specifically an explanatory design, and as such it aims to explore the "extent to which two variables co-vary, that is, where changes in one variable are reflected in the changes in the other" Creswell, 2008, p. 358). As an explanatory design, the data is collected at one time, and does not concern itself with past or future responses of participants. Two scores were collected from each participant with each score representing each variable of study (Creswell, 2008). To address the research questions in this study, the following statistical treatment were used: to examine the factor structure of personality disorders and measures of the five-factor model of personality, factor analysis was used.

To examine the links between personality disorder scales and the Personality disorder scales are assessed by use of the MCMI-III (Millon Clinical Multiaxial Inventory, Millon, Millon, and Davis, 1994) and the five factor model of personality is assessed using the NEO-PI R (NEO-PI-R, Neuroticism, Extraversion and Openness Personality Inventory - Revised, Costa \& McCrae, 1992), multiple regression was used.

Research Setting: Data collectors obtained data from different parts of the Philippines: some parts of Luzon (Manila and Quezon City); some parts of Visayas (Tacloban, Leyte) and Mindanao (Cagayan de Oro).

Sampling Procedure: Simple random sampling was used in this study. Several data collectors were involved in this study who gathered data from different parts of the country. The participants were 828 respondents from various areas in the Philippines. $35.5 \%$ males and $64.3 \%$ females. The average age of respondents is $20.29(\mathrm{SD}=4.83)$.

Data Gathering Technique: Two assessment measures were used for this study: the MCMI-III and the NEO PI R.

The Millon Clinical Multiaxial Inventory (MCMI-III, Millon, Millon, and Davis, 1994) consists of 175 true-false items from which scores on 14 personality disorders can be derived, as well as 10 clinical syndrome scales. The MCMI-III reflects explicitly the diagnostic criteria of the DSM-IV. The reported reliability coefficients of the MCMI-III ranged from .67 to .89 and the test-retest values ranged from .88 to .93 (Millon, et al., 1994). The MCMI-III is primarily used for differentiating psychiatric disorders and thus is not used to assess normal functioning adults and is not used as to assess general personality structure. However, evidence suggests that its validity is still maintained when used on non-clinical population. The study of Dyce, O'connor, Parkins and Janzen (1997) who examined the correlational structure of personality disorders scales with the MCMI-III found the correlation matrix of their non-clinical sample was highly similar to the 
correlations of Millon's (1994) clinical sample. This suggests a correlated nature of the personality disorders symptoms of the non clinical sample with the clinical sample. Studies had been conducted using non-clinical samples. For example, the study of ofAluja, Cuevas, and Garcia, (2007) which explored the relationship of Zuckerman personality model and the 14 personality disorder scales of the MCMI-III used subjects from the general population. In another study of the same authors, which examined the relationship of NEO-PI-R domains and facets with MCMI-III 14 personality scales, also used a non-clinical sample of university students in Madrid.

In the current study, the raw scores were used in the analysis, not base rate scores (BR). Base rate scores, which are basically ordinal-level classifications of the severity of traits or style or syndrome (Millon\& Bloom, 2008), reflect diagnoses of the individuals that consist the normative sample (Weiner and Craighead, 2010), and hence may not reflect Filipino clinical norms. Raw scores reflect the total of items endorsed in each personality disorder scale in the MCMI III

The second assessment measure used in the present study is the NEO-PI-R, Neuroticism, Extraversion and Openness Personality Inventory - Revised. This is a personality measure based on the Five-Factor Model that measures the interpersonal, motivational, emotional and attitudinal styles. It consists of 240 personality items with a 5-point scale response ranging from 0 (strongly disagree) to 4 (strongly agree). The internal consistency coefficients range from .86 to .95 for domain scales and .56 to .90 for facet scales (Costa \& McCrae, 1992).

\section{RESULTS}

\section{Factor Analyses}

To examine the factor structure of personality disorders and FFM, factor analysis was used. The Kaiser-Meyer-Olkin (KMO) measure of sampling adequacy was.93 which allowed for factor analysis to proceed. The Bartlett's Test of Sphericity (BTS) yielded an $\chi^{2}=17475.972, \mathrm{p}<.001$, demonstrating that the correlation matrix is not an identity matrix and thus further indicating that the data is factorable. Eight factors accounted for $56.831 \%$ of the total variance.

A principal components analysis and varimax rotation of the 30 NEO-PI-R facets and 14 MCMI-III personality disorder scales yielded eight factors according to the Eigenvalue $>1$ criteria.

The first factor grouped all most of the PD scales except Compulsive and Narcissistic, the NEO-PI-R depression facet in negative also loaded in this factor. Factor analytic results showed that most personality disorders, 10 of 12, with factor loadings higher than .40 loaded on the first factor. This factor may be considered as the "neuroticism" factor. Only the narcissistic and the compulsive scales did not load in this factor. This factor conceptually resembles Filipino identified social attribute as "dangerousness" (Imperio, Church, Katigbak, Reyes, 2008).
The second factor grouped the Neuroticism facets (N1 Anxiety, N5 Impulsiveness, and Depression N3); Extraversion facets (E2 Gregariousness and E3 Assertiveness); as well as Conscientiousness facet ( 2 2 Order) and Openness to Experience (O3 Feelings). The second factor, which includes anxiety, depression, impulsiveness, gregariousness, assertiveness, and openness to experience - ideas, may form as "extraversion" factor. An extraverted person is described as who prefers to be in social contact, who is forceful and open in his or her expressions of feelings as anxiety and depression. This factor is conceptually similar to "prominence" (Imperio et al., 2008).

On the third factor, three of Conscientiousness facets were loaded (C5 Self-discipline, C6 Deliberation and C1 Competence). The Neuroticism facet (N6) Vulnerability to stress also loaded in this factor, as well as the openness to experience facet (O5 Ideas). Conscientiousness factor is integrated in the third factor by competence, self-discipline, and deliberation, vulnerability, and openness to experienceideas Filipinos may consider a conscientious individual as one who can intelligently, deliberately plan for and effectively work to achieve his goals, but who may be susceptible to stress. This factor bears some conceptual resemblance to the Filipino social attribute "respectability" (Imperio et al., 2008).

The fourth factor is composed of Extraversion facets (E1) Warmth, (E5) Excitement, and (E4) Activity; Agreeableness facet (A1) Trust is also grouped in this factor, as well as PD scale Histrionic. The fourth factor may be described as the "agreeableness" factor, preference for interactions which includes warmth or those that are friendly; activity and excitement-seeking, or those that are interesting, and trust, those based on which are sincere and well-intentioned.

The fifth factor grouped PD scale Compulsive and Antisocial in negative; and two Conscientiousness facets (C4 Achievement Striving and C3 Dutifulness), and a lone Openness to Experience facet (O3 Actions). The fifth factor reflects extent of organization, persistence, and control in goal-oriented behaviors, could also be considered a conscientiousness factor includes PD scales antisocial in negative and compulsive, openness to experience-actions, dutifulness and achievement-striving. This factor closely resembles Filipino attribute "presentableness" (Imperio et al., 2008).

Two Agreeableness facets (A3 Altruism and A5 Modesty); two Openness to Experience facets (01 Fantasy and O6 Values); as well Conscientiousness facet C1 Competence loaded on the sixth factor. The seventh factor loaded Openness to Experience facet $\mathrm{O} 2$ Aesthetics and Agreeableness facet A6 Tender mindedness. Factor six integrated the scales openness to experience scale (fantasy and values) and agreeableness (altruism and tender mindedness).Openness to experience was formed in factor seventh includes aesthetics, the appreciation of art and beauty, and tender mindedness. Taken together, these facets are 
conceptually similar to the Filipino attribute "charisma" (Imperio et al., 2008).

The eighth factor is composed of PDs Histrionic and Narcissistic. The last factor includes two of cluster B personality disorders: histrionic and narcissistic are conceptually related to Filipino attributes "attractive" and "destructive" (Imperio et al., 2008). Those that did not load in any factor are compliance (A4), straightforwardness (A2); positive emotions (E6), angry-hostility (N2) and Selfconsciousness (N4).

\section{Correlational Analyses}

The relationship among the five NEO domains and personality disorders are shown in Table 2. This table shows that the five factor model is modestly related to personality disorders as described in the DSM IV. For example, PDs such as schizoid, avoidant, depressive, antisocial, sadistic, negativistic, schizotypal, borderline, masochistic and paranoid are significantly, modestly related to all FFM dimensions. This table also reveals that only a few FFM dimensions do not correlate with PDs: For example, dependent PD is not significantly related to openness to experience; histrionic PD is not significantly associated with openness to experience, agreeableness and conscientiousness; and narcissistic PD is not related to neuroticism, extraversion and conscientiousness. The correlations between FFM and PD are shown in this table 2 in the appendix to be substantial reflecting shared FFM dimensions in PD suggesting that the five factors and its facets can be used to predict personality disorder.

\section{DISCUSSION}

As the current study is exploratory in nature, the results here maintain the eight factors uncovered in the analyses. As an exploratory study it does not then seek to confirm the fivepersonality factors outlined by the five-factor model. The cultural differences between this study's research participants and the normative sample merits the use of exploratory approach. The existing differences in the socio-cultural context, such as language, manner of response to direct questioning in the instruments, openness in self-rating, and orientation, as in collectivistic or individualistic, suggest thatit may not be best to exclude the investigation of additional factors revealed in the current analyses(Robins, Fraley, Krueger, 2009). Considering these cultural differences, the exploratory nature of the current study examined the variables of interest here in general and nonspecialized terms without any attempt to restrict the description of those variables in question.

The correlations between FFM and PD are shown in the Table 2, appended, to be substantial reflecting shared FFM dimensions in PD suggesting that the five factors and its facets can be used to predict personality disorder. This result is supported by the study of DeFruyt, De Clercq, Miller, Rolland, Jung, Taris, Furnham, \& Van Hiel (2009) demonstrated that personality disorders can be estimated from NEO PI-R.

Other researchers argue that personality disorders can be thought of as extreme values on one or more of the five personality dimensions (Piedmont, Sherman, Sherman, DyLiacco, and Williams, 2009). For example, extreme scores on the personality dimension openness to experience as measured by Experiential Permeability Inventory is related to preoccupation with fantasy, unstable aims, nonconformity to social behavioral conventions, eccentricity and diffused selfconcept. These tendencies of excessive openness are related schizotypal personality disorder (Piedmont, et al., 2009).

The same table shows that most PDs except narcissistic are significantly related to personality domain Neuroticism (N). This suggests that the lack of emotional stability expressed as anger, anxiety, depression and vulnerability, is the manifest symptom of personality disorder. The study of Aluja, Cuevas, Garcia, \& Garcia (2007)supports this conclusion whose study revealed that neuroticism as the strongest predictor of personality disorders

Kotov, Gamez, Schmidt, and Watson (2010) found that common mental disorders are significantly related to personality and have similar trait profiles. Their results confirmed the results for this study in that theirs have shown that neuroticism was the strongest correlate across the board.

Table 3, appended, presentsthe correlations between PD scales and NEO PI -R facets. The results of this study show a modest correspondence between personality disorder and personality trait facets.

For example, schizoid PD is shown to be significantly related to all NEO PI-R facets except warmth (E1), excitementseeking (E5), openness to ideas (O5), dutifulness (C3) and deliberation (C6); depressive PD is related to all facets except openness to ideas (O5) and dutifulness (C3); antisocial PD is related to all but achievement-striving (C4); sadistic PD is significantly associated with all with the exception of dutifulness (C3); and schizotypal is not related to openness to ideas (O5) and dutifulness (C3).

These results suggest that there is conceivably a five-factor profile for each personality disorder. This conclusion was supported by the study of Saulsman and Page (2004) which revealed that each $\mathrm{PD}$ displays a five-factor model profile that is meaningful and predictable.

The same table also shows that more than one NEO facet is significantly associated with PD. This suggests that the expression of PD may take the form of a profile than a one-toone correspondence of PD and NEO-facet scales. Facet scales that did not show significant relationship with PD may suggest that these facet scales are not salient to a particular PD description.

The results of this study is consistent with the study of Rosellini and Brown (2011) which showed that neuroticism is 
a significant, positive correlate with DSM IV anxiety disorders.

Widiger (2011) showed the link between personality and psychopathology in three ways, namely: pathoplastic relationship or the manner personality and psychopathology influence the presentation of one another; spectrum relationship or the sharing of common and fundamental etiology; and both can have a causal role in the development of one another.

Widiger (2011) suggest that the relationship of personality and pathology is pathoplastic. The pathoplastic relationship of personality and pathology is described as bidirectional, in that, a person's premorbid personality traits can affect with pathological symptoms; and the appearance of clinical symptoms may be affected by given personality traits. Widiger (2011) continues to argue that the relationship of personality and psychopathology may be made more complex by the possibility that they may not be distinct constructs but exist along a common continuum of functioning. Personality disorders are thought be deviations of basic personality traits, and personality disorders are could be onset of mental disorders. It is also argued that the causal relationship of personality and pathology is bidirectional: mental disorder can contribute to personality changes, and personality can contribute to the development of pathology.

Table 4, appended, shows the results of the regression analyses conducted to establish the relationship between each of the NEO domains and facets to personality dimensions and to examine the variance of each personality disorder explained by the NEO domains and its facets.

It can be seen than all NEO domains; neuroticism, extraversion, openness to experience, agreeableness, and conscientiousness make significant contributions to the variance of personality disorders. For example, the NEO domains contribute 16 percent of the variance of antisocial PD, 21 percent of avoidant PD, 20 percent of borderline, 27 percent of dependent and depressed PD, 10 percent of histrionic, and 11 percent of narcissistic.

However, when facet scores were added to the equation, the variance explained for PD is doubled. For example, with facet scores included, the variance explained for antisocial PD improved to 32 percent, 34 percent for avoidant PD, 35 percent for borderline, 37 percent for compulsive PD, 34 percent for depressed and dependent, 29 percent for histrionic.

The present study is confirmed by the study of Nestadt, Costa, Hsu, Samuels, Bienvenu, and Eaton (2008) which demonstrated the NEO domain and facet scores partially explained the variance in PD dimensions. Additionally, Reynolds and Clark (2001) reported that the NEO facets allowed for substantial improvement than when only the domains were used in the equation in predicting interview -based ratings of DSM IV personality disorders. Collectively, the five NEO domains explained each of personality disorder moderately, and the inclusion of the facet scores improved the degree to which the NEO dimensions explained personality disorder.

Overall, the NEO domains are able to explain a fifth or a third of personality disorder. This percent of the variance explained by the NEO is lower than that what was previously reported in other studies. The study of O'Connor and Dyce reported that the NEO explained 46 percent of the variance of personality disorders among college students who were assessed using the MCMI - III.

The qualification of PDs then needs to be assessed in relation to the individual's cultural experiences.

While the results of this study showed significant correspondence between general personality and personality disorder, the correlations are shown here to be low to moderate. The significant correlation between personality and PD may suggest that the NEO may be an appropriate medium for examining the relationship between normal and pathological personality traits. However, the low to moderate correlations of PD and personality dimensions it seems then necessary to use additional clinical material, beyond the NEO, to describe personality disorder dimensions.

Although the FFM provides a general overview to the understanding of personality; it may be premature to commit to this one model to the exclusion of others. One reason for not adhering to this model while ruling out others is that in this study FFM explains less of the variance than is typical studies done with undergraduates done in the west (Barr, 1997; Waldman, Atwater, \& Davidson, 2004). This may be attributed to the research participants' responses as speciestypical reflexes (Burger, 1997). In the context of culture, these may be thought of as behaviors commonly engaged in by members of a given culture, an in the Filipino culture in this study. In addition, theoretical reasons suggest that measures used may not have captured the personality traits that occur in the natural Filipino language (Millon, 2003) or that traits assessed in the measures are not linked to national personality stereotypes (Terraciano\& McCrae, 2006).

From the results of this study, it could also be assumed that a comprehensive Filipino model of personality may consist of more than five factors. For example, Church, Katigbak, and Reyes, (1996) uncovered a 13 category-system. In 1998, further exploration of the Filipino structure of the same authors revealed 7 personality dimensions while Mallare's (2007) lexical analysis revealed six structures. The most current lexical study of Filipino descriptors by Imperio et al. (2008) identified ten dimensions of physical and social attributes. The evidence then from Filipino personality structure analysis appears to almost always yield more than five factors. Katigbak, Church, Guanzon-Lapeña, Carlota and del Pilar (2002) concluded that a minority of indigenous constructs are less well accounted for by the FFM and that these constructs are not unheard of in Western cultures, but 
may be specifically prominent or arranged differently in the Filipino culture.

In conclusion, this study provides measured support to the correspondence of personality disorders and FFM theory of personality with each PD significantly associated with personality domains and facets contribute significantly to personality disorder variance.

\section{SUMMARY, CONCLUSION, AND RECOMMENDATION}

The present study examined the factor structure of personality disorders and personality traits in terms of NEO domains and facets. Eight factors were extracted usingvarimax components analysis. Using the terms proposed by Imperio et al. (2008), factor 1 is labeled as "dangerousness", factor 2 as "prominence", factor 3 as "respectability", factor 4 as "agreeableness", factor 5 as "presentableness", merged factors 6 and 7 as "charisma", and factor 8 as "attractive/destructive" factor.It is then reasonable to assume that the descriptions generated from the factor analysis are conceptually appropriate when applied in the Filipino cultural setting.

As recommendation, this study may be replicated using the DSM 5 framework of describing personality disorders. In the DSM 5 framework, the approach is considered to be dimensional descriptive. In the use of this current framework of describing pathology, a measure of personality disorders that reflects DSM 5 PD description may have to be used.

\section{APPENDICES}

Table 1: Factor Loadings Based on Principal Components Analysis of MCMI-III And NEO-PI-R Personality Scales In Filipino Sample

\begin{tabular}{|c|c|c|c|c|c|c|c|c|}
\hline & I & II & III & IV & V & VI & VII & VIII \\
\hline Schizoid & .710 & & & & & & & \\
\hline Avoidant & .823 & & & & & & & \\
\hline Depressive & .856 & & & & & & & \\
\hline Histrionic &.-441 & & & & & & & .838 \\
\hline Narcissistic & & & & & & & & .481 \\
\hline Antisocial & .629 & & & & -.401 & & & \\
\hline Sadistic & .723 & & & & & & & \\
\hline Compulsive & & & & & .753 & & & \\
\hline Negativistic & .839 & & & & & & & \\
\hline Masochistic & .844 & & & & & & & \\
\hline Schizotypal & .857 & & & & & & & \\
\hline Paranoid & .807 & & & & & & & \\
\hline N1: Anxiety & & .603 & & & & & & \\
\hline \multicolumn{9}{|l|}{ N2: Angry Hostility } \\
\hline N3: Depression & .444 & .454 & & & & & & \\
\hline \multicolumn{9}{|l|}{$\begin{array}{c}\text { N4: Self- } \\
\text { consciousness }\end{array}$} \\
\hline N5: Impulsiveness & & .463 & & & & & & \\
\hline N6: Vulnerability & & & .637 & & & & & \\
\hline E1: Warmth & & & & .715 & & & & \\
\hline E2: Gregariousness & & .602 & & & & & & \\
\hline E3: Assertiveness & & .423 & & & & & & \\
\hline E4: Activity & & & & .411 & & & & \\
\hline $\begin{array}{l}\text { E5: Excitement- } \\
\text { seeking }\end{array}$ & & & & .544 & & & & \\
\hline \multicolumn{9}{|l|}{$\begin{array}{l}\text { E6: Positive } \\
\text { emotions }\end{array}$} \\
\hline O1: Fantasy & & & & & & .478 & & \\
\hline O2: Aesthetics & & & & & & & .600 & \\
\hline O3: Feelings & & .451 & & & & & & \\
\hline O4: Actions & & & & & .476 & & & \\
\hline
\end{tabular}




\begin{tabular}{|c|c|c|c|c|c|c|c|}
\hline O5: Ideas & & .582 & & & & & \\
\hline O6: Values & & & & & .419 & & \\
\hline A1: Trust & & & .422 & & & & \\
\hline $\begin{array}{c}\mathrm{A} 2: \\
\text { Straightforwardness }\end{array}$ & & & & & & & \\
\hline A3: Altruism & & & & & .584 & & \\
\hline A4: Compliance & & & & & & & \\
\hline A5: Modesty & & & & & .541 & & \\
\hline $\begin{array}{l}\text { A6: Tender } \\
\text { mindedness }\end{array}$ & & & & & & .553 & \\
\hline $\mathrm{C} 1$ : Competence & & .435 & & & & & \\
\hline C2: Order & .465 & & & & & & \\
\hline C3: Dutifulness & & & & .428 & & & \\
\hline $\begin{array}{l}\text { C4: Achievement } \\
\text { Striving }\end{array}$ & & & & .579 & & & \\
\hline C5: Self-discipline & & .488 & & & & & \\
\hline C6: Deliberation & & .475 & & & & & \\
\hline
\end{tabular}

Table 2: Correlations between NEO dimensions and PD

\begin{tabular}{|c|c|c|c|c|c|c|c|c|c|c|c|c|c|c|}
\hline & $\begin{array}{c}\text { Schiz } \\
\text { oid }\end{array}$ & $\begin{array}{c}\text { Avoida } \\
\text { nt }\end{array}$ & $\begin{array}{l}\text { Depresss } \\
\text { ive }\end{array}$ & $\begin{array}{c}\text { Depend } \\
\text { ent }\end{array}$ & $\begin{array}{c}\text { Histrioni } \\
\mathrm{c}\end{array}$ & $\begin{array}{c}\text { Narcissi } \\
\text { stic }\end{array}$ & $\begin{array}{c}\text { Anti } \\
\text { social }\end{array}$ & Sadistic & Compulsive & Negativistic & $\begin{array}{l}\text { Masochis } \\
\text { tic }\end{array}$ & $\begin{array}{c}\text { Schizo } \\
\text { typal }\end{array}$ & $\begin{array}{l}\text { Border } \\
\text { line }\end{array}$ & Paranoid \\
\hline $\begin{array}{c}\text { Neurotici } \\
\text { sm }\end{array}$ & $340 * *$ & $.424 * *$ & $464 * *$ & $.456^{* *} *$ & $-.167 * *$ & -.006 & $.353^{* *}$ & $.436^{* *}$ & $.461 * *$ & $.461 * *$ & $.496^{* *}$ & $.454 * *$ & $445^{* *}$ & $.405 * *$ \\
\hline $\begin{array}{l}\text { Extravers } \\
\text { ion }\end{array}$ & $\begin{array}{c}.212^{*} \\
*\end{array}$ & $.247 * *$ & $.334 * *$ & $.412 * *$ & $.079 *$ & .124 & $.343 * *$ & $.360 * *$ & $.365^{* *} *$ & $.365 * *$ & $.366^{*}$ & $.321 * *$ & $.369 * *$ & $.271 * *$ \\
\hline $\begin{array}{l}\text { Openness } \\
\text { to } \\
\text { Experien } \\
\text { ce }\end{array}$ & $\begin{array}{c}.174 * \\
*\end{array}$ & $.149 * *$ & $.202 * *$ & .102 & .031 & $.163^{* *}$ & $.137 * *$ & $.224 * *$ & $.207 * *$ & $.207 * *$ & $.210^{*}$ & $.240 * *$ & $.186^{* * *}$ & $.273 * *$ \\
\hline $\begin{array}{c}\text { Agreeabl } \\
\text { eness }\end{array}$ & $\begin{array}{c}.163^{*} \\
*\end{array}$ & $.145^{* *}$ & $.232 * *$ & $.243 * *$ & .015 & $.140 * *$ & $.249 * *$ & $.326^{* *}$ & $.272 * *$ & $.272 * *$ & $.259 * *$ & $.244 * *$ & $.253^{* *}$ & $.261 * *$ \\
\hline $\begin{array}{l}\text { Conscien } \\
\text { tiousness }\end{array}$ & $\begin{array}{c}.208^{*} \\
*\end{array}$ & $.237 * *$ & $.285^{* *}$ & $.229 * *$ & -.057 & .010 & $.205^{* *}$ & $.347 * *$ & $.317 * *$ & $.317 * *$ & $.282 * *$ & $.294 * *$ & $.272 * *$ & $.338 * *$ \\
\hline
\end{tabular}

$$
\begin{aligned}
& * * \mathrm{p}<.05 \\
& . * \mathrm{p}<.05
\end{aligned}
$$

\begin{tabular}{|c|c|c|c|c|c|c|c|c|c|c|c|c|c|c|}
\hline & Schizoid & Avoidant & Depressive & Dependent & Histrionic & Narcissistic & $\begin{array}{c}\text { Anti } \\
\text { Social }\end{array}$ & Sadistic & Compulsive & Negative & Masochistic & $\begin{array}{c}\text { Schizo } \\
\text { Typal }\end{array}$ & $\begin{array}{l}\text { Border } \\
\text { Line }\end{array}$ & Paranoid \\
\hline $\mathrm{N} 1$ & $0.223^{* *}$ & $0.263^{* *}$ & $0.276^{* *}$ & $0.316^{* *}$ & -0.051 & -0.004 & $0.200^{* *}$ & $0.257^{* *}$ & $-0.094 * *$ & $0.251^{* *}$ & $0.289^{* *}$ & $0.271^{* *}$ & $0.254 * *$ & $0.222 * *$ \\
\hline $\mathrm{N} 2$ & $0.283^{* *}$ & $0.352 * *$ & $0.309^{* *}$ & $0.237^{* *}$ & $-0.247 * *$ & -0.036 & $0.238^{* *}$ & 0.341 ** & $-0.117 * *$ & $0.351^{* *}$ & $0.360 * *$ & 0.339 ** & $0.311^{* *}$ & $0.358 * *$ \\
\hline N3 & $0.339 * *$ & $0.453^{* *}$ & $0.501 * *$ & $0.504 * *$ & $-0.261 * *$ & $-0.165 * *$ & 0.360 ** & $0.362 * *$ & $-0.310 * *$ & $0.446^{* *}$ & $0.513 * *$ & $0.439 * *$ & 0.467 ** & $0.344 * *$ \\
\hline $\mathrm{N} 4$ & $0.213 * *$ & $0.282^{* *}$ & 0.319 ** & $0.333 * *$ & $-0.156^{* *}$ & $-0.106^{* *}$ & $0.192^{* *}$ & 0.241 ** & $-0.117 * *$ & $0.275^{* *}$ & $0.342 * *$ & $0.279 * *$ & $0.281^{* * *}$ & $0.218^{* *}$ \\
\hline N5 & $0.214 * *$ & $0.228 * *$ & $0.250^{* * *}$ & $0.286^{* *}$ & -0.022 & $0.093 * *$ & $0.272 * *$ & $0.323 * *$ & $-0.145^{* *}$ & $0.289 * *$ & $0.247 * *$ & $0.245^{* *}$ & $0.279 * *$ & $0.215^{* *}$ \\
\hline N6 & $0.093^{* *}$ & $0.094 * *$ & $0.101 * *$ & $0.137^{* *}$ & 0.015 & $0.077 * *$ & $0.119^{* *}$ & $0.152 * *$ & 0.007 & $0.153^{* *}$ & $0.127 * *$ & $0.142^{* *}$ & $0.116^{* *}$ & $0.140 * *$ \\
\hline E1 & -0.009 & 0.014 & $0.170^{* * *}$ & 0.241 ** & $0.257 * *$ & $0.124 * *$ & $0.174^{* *}$ & $0.163^{* *}$ & -0.045 & $0.152 * *$ & $0.177 * *$ & $0.104 * *$ & $0.185 * *$ & $0.097 * *$ \\
\hline E2 & $0.344 * *$ & $0.319 * *$ & $0.285^{* *}$ & $0.319^{* *}$ & $-0.207 * *$ & -0.006 & $0.215^{* *}$ & $0.255^{* *}$ & $-0.078 * *$ & $0.288^{* *}$ & $0.294 * *$ & $0.298 * *$ & $0.270^{* * *}$ & $0.244 * *$ \\
\hline E3 & $0.328 * *$ & $0.330 * *$ & $0.404 * *$ & $0.377^{* *}$ & $-0.143 * *$ & 0.043 & $0.341^{* *}$ & $0.404 * *$ & $-0.202 * *$ & $0.397 * *$ & $0.393 * *$ & $0.392 * *$ & $0.429 * *$ & $0.353 * *$ \\
\hline $\mathrm{E} 4$ & $0.155^{* *}$ & $0.153^{* *}$ & $0.246^{* *}$ & $0.209^{* *}$ & 0.067 & 0.197 ** & $0.223 * *$ & $0.291 * *$ & -0.002 & $0.278^{* *}$ & $0.250 * *$ & $0.278 * *$ & 0.250 ** & $0.295 * *$ \\
\hline ES & -0.052 & 0.004 & $0.105^{* *}$ & $0.179^{* *}$ & $0.239 * *$ & $0.154 * *$ & $0.263^{* *}$ & $0.182 * *$ & $-0.145^{* *}$ & $0.182 * *$ & $0.118^{* *}$ & $0.117^{* *}$ & $0.198^{* *}$ & $0.113 * *$ \\
\hline
\end{tabular}

TABLE 3: Correlations between PD scales and N 
International Journal of Research and Innovation in Social Science (IJRISS) |Volume V, Issue V, May 2021|ISSN 2454-6186

\begin{tabular}{|c|c|c|c|c|c|c|c|c|c|c|c|c|c|c|}
\hline E6 & $0.098^{* *}$ & $0.068^{* *}$ & $0.150^{* *}$ & $0.140^{* *}$ & $0.094 * *$ & $0.099^{* *}$ & $0.187^{* *}$ & $0.217 * *$ & -0.061 & $0.202 * *$ & $0.155^{* *}$ & $0.153^{* *}$ & $0.161^{* *}$ & $0.148^{* *}$ \\
\hline O1 & $0.204^{* *}$ & $0.159^{* *}$ & $0.157^{* *}$ & 0.065 & 0.004 & $0.154^{* *}$ & $0.095^{* *}$ & $0.166^{* *}$ & $0.135^{* *}$ & $0.121^{* *}$ & $0.188^{* *}$ & $0.190^{* *}$ & $0.115^{* *}$ & $0.218^{* *}$ \\
\hline $\mathrm{O} 2$ & $0.097 * *$ & $0.076^{* *}$ & $0.152^{* *}$ & $0.098^{* *}$ & 0.013 & $0.094^{* *}$ & $0.145^{* *}$ & $0.161^{* *}$ & -0.033 & $0.183^{* *}$ & $0.157^{* *}$ & $0.176^{* *}$ & $0.149 * *$ & $0.165^{* *}$ \\
\hline $\mathrm{O} 3$ & $0.220 * *$ & $0.245^{* *}$ & $0.288^{* *}$ & $0.263^{* *}$ & -0.040 & 0.065 & $0.202 * *$ & $0.244 * *$ & -0.051 & $0.271 * *$ & $0.315^{* *}$ & $0.292 * *$ & $0.288 * *$ & $0.255^{* *}$ \\
\hline $\mathrm{O} 4$ & $0.121^{* *}$ & $0.127^{* *}$ & $0.123^{* *}$ & $0.160^{* *}$ & $0.077 * *$ & $0.101 * *$ & $0.071^{* *}$ & $0.161^{* *}$ & $0.149 * *$ & $0.154 * *$ & $0.153 * *$ & $0.174 * *$ & $0.149 * *$ & $0.195^{* *}$ \\
\hline O5 & 0.001 & $-0.024 * *$ & 0.019 & -0.048 & 0.056 & $0.095^{* *}$ & $0.105^{* *}$ & $0.084^{* *}$ & -0.005 & 0.045 & -0.012 & 0.040 & 0.061 & 0.063 \\
\hline O6 & $0.174 * *$ & $0.166^{* * *}$ & $0.175^{* *}$ & $0.119^{* *}$ & -0.026 & $0.089 * *$ & $0.086^{* *}$ & $0.177 * *$ & 0.044 & $0.190^{* *}$ & $0.216^{* * *}$ & $0.191 * *$ & $0.126^{* *}$ & $0.223 * *$ \\
\hline $\mathrm{A} 1$ & $0.133^{* *}$ & $0.152^{* * *}$ & $0.223^{* *}$ & $0.242^{* *}$ & 0.031 & $0.087^{* *}$ & $0.092^{* *}$ & $0.205^{* *}$ & $0.072 * *$ & $0.199 * *$ & $0.234 * *$ & $0.198^{* *}$ & $0.172 * *$ & $0.200 * *$ \\
\hline A2 & $0.194 * *$ & $0.175^{* * *}$ & $0.212^{* *}$ & $0.183^{* *}$ & -0.036 & $0.216^{* *}$ & $0.322 * *$ & $0.390^{* *}$ & $-0.193^{* *}$ & $0.320^{* *}$ & $0.221 * *$ & $0.264 * *$ & $0.290 * *$ & $0.326^{* *}$ \\
\hline A3 & $0.098^{* *}$ & $0.117^{* *}$ & $0.139 * *$ & $0.092^{* *}$ & -0.008 & $0.099 * *$ & $0.104 * *$ & $0.218^{* *}$ & 0.015 & $0.153^{* *}$ & $0.156^{* *}$ & $0.142^{* *}$ & $0.131 * *$ & $0.173 * *$ \\
\hline A4 & 0.198 ** & $0.153^{* * *}$ & $0.252^{* *}$ & $0.258^{* *}$ & -0.013 & $0.113^{* *}$ & $0.367^{* *}$ & $0.342^{* *}$ & $-0.257 * *$ & $0.285^{* *}$ & $0.277 * *$ & $0.277^{* *}$ & $0.315^{* *}$ & $0.246^{* *}$ \\
\hline A5 & $0.096^{* *}$ & 0.058 & $0.090^{* *}$ & $0.076^{* *}$ & -0.015 & $0.159 * *$ & $0.110^{* *}$ & $0.163^{* *}$ & -0.007 & $0.108^{* *}$ & $0.109 * *$ & $0.128 * *$ & $0.092 * *$ & $0.144 * *$ \\
\hline A6 & $0.070^{* *}$ & $0.122^{* * *}$ & $0.138^{* *}$ & $0.167^{* *}$ & -0.017 & $0.069 * *$ & $0.144 * *$ & $0.171^{* *}$ & $-0.082^{* *}$ & $0.203^{* *}$ & $0.187 * *$ & $0.149 * *$ & $0.189 * *$ & $0.169 * *$ \\
\hline $\mathrm{C} 1$ & $0.111^{* *}$ & $0.081^{* * *}$ & $0.123^{* *}$ & $0.089^{* *}$ & -0.017 & $0.195^{* * *}$ & $0.197^{* *}$ & $0.235^{* *}$ & -0.009 & $0.194 * *$ & $0.150^{* * *}$ & $0.143^{* *}$ & $0.159^{* *}$ & $0.205^{* *}$ \\
\hline $\mathrm{C} 2$ & $0.163^{* *}$ & $0.171^{* *}$ & $0.202^{* *}$ & 0.221 & -0.063 & -0.031 & $0.226^{* *}$ & $0.166^{* * *}$ & $-0.206^{* *}$ & $0.234 * *$ & $0.268 * *$ & $0.195^{* *}$ & $0.226^{* *}$ & $0.156^{* *}$ \\
\hline $\mathrm{C} 3$ & -0.019 & -0.001 & -0.024 & -0.050 & 0.014 & 0.054 & $\begin{array}{c}- \\
0.113^{* *} \\
\end{array}$ & 0.029 & $0.306^{* *}$ & -0.033 & -0.056 & -0.008 & $\begin{array}{c}- \\
0.087 * * \\
\end{array}$ & 0.045 \\
\hline $\mathrm{C} 4$ & $0.088 * *$ & $0.119^{* *}$ & $0.128^{* *}$ & $0.089^{* *}$ & 0.061 & $0.178^{* *}$ & -0.027 & $0.160 * *$ & $0.290^{* *}$ & $0.107^{* *}$ & $0.102 * *$ & $0.130 * *$ & 0.067 & $0.184 * *$ \\
\hline C5 & $0.195^{* *}$ & $0.202^{* *}$ & $0.223^{* *}$ & $0.238^{* *}$ & -0.032 & $0.138^{* *}$ & $0.215^{* *}$ & $0.281^{* *}$ & -0.007 & $0.266^{* *}$ & $0.247 * *$ & $0.252 * *$ & $0.242 * *$ & $0.263^{* *}$ \\
\hline C6 & 0.067 & 0.033 & $0.116^{* *}$ & $0.069^{* *}$ & $0.083^{* *}$ & $0.154 * *$ & $0.076^{* *}$ & $0.201^{* *}$ & $0.122 * *$ & $0.159^{* *}$ & 0.070 ** & $0.094 * *$ & $0.124 * *$ & $0.180 * *$ \\
\hline
\end{tabular}

${ }^{* *} \mathrm{p}<.05$

Table 4: Regression coefficients for FFM personality dimensions predicting personality disorder

\begin{tabular}{|c|c|c|c|c|c|c|c|c|c|c|c|c|c|}
\hline & $\begin{array}{l}\text { Anti } \\
\text { social }\end{array}$ & Avoidant & $\begin{array}{c}\text { Borderlin } \\
\mathrm{e}\end{array}$ & Compulsive & Dependent & Depressed & $\begin{array}{l}\text { Histrio } \\
\text { nic }\end{array}$ & $\begin{array}{l}\text { Narcissis } \\
\text { tic }\end{array}$ & Negative & Paranoid & Sadistic & Schizoid & Schizotypal \\
\hline $\mathrm{N}$ & .296 & .604 & .401 & -.440 & .515 & .522 & -.503 & -.456 & .373 & .291 & .225 & .414 & .476 \\
\hline $\mathrm{E}$ & .192 & .017 & .179 & -.056 & .212 & .104 & .264 & .188 & .121 & .066 & .127 & .029 & .094 \\
\hline $\mathrm{O}$ & -.033 & .006 & .006 & .214 & -.105 & .022 & .103 & .108 & .001 & .093 & -.058 & .059 & .072 \\
\hline $\mathrm{A}$ & .049 & -.160 & -.060 & -.096 & -.025 & -.085 & .086 & .165 & -.044 & -.024 & .073 & -.079 & -.074 \\
\hline$r$ & .399 & .454 & .441 & .379 & .518 & .481 & .321 & .328 & .422 & .418 & .388 & .352 & .467 \\
\hline$r^{2}$ & .159 & .207 & .195 & .144 & .269 & .269 & .103 & .107 & .178 & .175 & .150 & .124 & .218 \\
\hline${ }^{a} r^{2}$ & .324 & .339 & .354 & .368 & .344 & .344 & .293 & .221 & .341 & $/ 221$ & .329 & .283 & .327 \\
\hline
\end{tabular}

** $\quad \mathrm{p}<.05$

${ }^{a} r^{2}$ with facet scale scores added

\section{REFERENCES}

[1] Aluja A., Cuevas, L., Garcia, L. \& Garcia, O. (2007). Predictions of the MCMI-III personality disorders from NEO-PI R domains and facets: Comparison between American and Spanish samples. International Journal of Clinical and Health Psychology, $v 7$.

[2] American Psychiatric Association: Diagnostic and Statistical Manual of Mental Disorders, Fourth Edition, Text Revision. Washington, DC, American Psychiatric Association, 2000.

[3] Creswell, J. (2008). Educational research: Planning, conducting, and evaluating quantitative and qualitative research. New Jersey: Pearson: Merrill Prentice Hall
[4] Costa, P. \&Widiger, T. (2002).Personality disorders and the fivefactor model of personality. American Psychological Association.

[5] Costa, P., \&Widiger, T. (2002). Personality disorders and the fivefactor model of personality (2nd ed.). Washington, DC US: American Psychological Association. doi:10.1037/10423-000.

[6] De Fruyt, F., De Clercq, B. J., Miller, J., Rolland, J., Jung, S., Taris, R., Furnham, A., \& Van Hiel, A. (2009). Assessing personality at risk in personnel selection and development. European Journal of Personality, 23, 51-69.

[7] Dyce, J. A., O'Connor, B. P., Parkins, S. Y., \& Janzen, H. L. (1997). Correlational structure of the MCMI-III personality 
disorder scales and comparisons with other data sets. Journal of Personality Assessment, 69(3), 568-582.

[8] Kotov, R., Gamez, W., Schmidt, F., \& Watson, D. (2010). Linking "big" personality traits to anxiety, depressive, and substance use disorders: a meta-analysis. Psychological bulletin, 136(5), 768

[9] Imperio, S., Church, A., Katigbak, M., \& Reyes, J. (2008). Lexical studies of Filipino person descriptors: Adding personality-relevant social and physical attributes. European Journal of Personality, 22(4), 291-321.doi:10.1002/per.673.

[10] McCrae, R. R. \& Costa, P. T. (1990). Personality in adulthood. New York: The Guildford Press

[11] Millon,T., Millon, C., Grossman, S., \& Meagher, S. (2004). Personality disorders in modern life. New Jersey: John Wiley and Sons.

[12] Millon, T., \& Davis, R. D. (1997). The MCMI--III: present and future directions. Journal of personality assessment, 68(1), 69-85.

[13] Mondak, J. J. (2010). Personality and the foundations of political behavior.Cambridge University Press.

[14] Oliver, J. ,Angleitner, A., \&Ostendorf, F. (1988). The lexical approach to personality: A historical review of trait taxonomic research. European Journal of Personality v2.DOI: 10.1002/per.2410020302

[15] Piedmont, R. L., Sherman, M. F., Sherman, N. C., Dy-Liacco, G. S., \& Williams, J. E. G. (2009). Using the five-factor model to identify a new personality disorder domain: The case for experiential permeability. Journal of Personalityand Social Psychology, 96, 1245-1258.
[16] Reynolds, S.\& Clark, L. (2001). Predicting dimensions of personality disorder from domains and facets of the five factor model. Journal of Personality v69(2). DOI: 10.1111/14676494.00142

[17] Robins, R. W., Fraley, R. C., \& Krueger, R. F. (Eds.). (2009). Handbook of research methods in personality psychology. Guilford Press.

[18] Rosellini, A. J., \& Brown, T. A. (2011). The NEO Five-Factor Inventory: latent structure and relationships with dimensions of anxiety and depressive disorders in a large clinical sample. Assessment, 18(1), 27-38.

[19] Sharifi, A. A., MOULAVI, H., \&Namdari, K. (2008). The validity of MCMI-III (Millon, 1994) scales.

[20] Saulsman, L., \& Page, A. (2004). The five-factor model and personality disorder empirical literature: A meta-analytic review. Clinical Psychology Review, 23(8), 1055-1085. doi:10.1016/j.cpr.2002.09.001.

[21] Weiner, I. B., \& Craighead, W. E. (Eds.). (2010). The Corsini Encyclopedia of Psychology, Volume 4 (Vol. 4).John Wiley \& Sons.

[22] Widiger, T. A. (2011).Personality and psychopathology. World Psychiatry,10(2), 103-106 